\title{
Experimental Observation of Optically Trapped Atoms
}

\author{
Steven Chu, J. E. Bjorkholm, A. Ashkin, and A. Cable \\ AT\&T Bell Laboratories, Holmdel, New Jersey 07733 \\ (Received 14 April 1986)
}

\begin{abstract}
We report the first observation of optically trapped atoms. Sodium atoms cooled below $10^{-3} \mathrm{~K}$ in "optical molasses" are captured by a dipole-force optical trap created by a single, strongly focused, Gaussian laser beam tuned several hundred gigahertz below the $D_{1}$ resonance transition. We estimate that about 500 atoms are confined in a volume of about $10^{3} \mu \mathrm{m}^{3}$ at a density of $10^{11}-10^{12}$ $\mathrm{cm}^{-3}$. Trap lifetimes are limited by background pressure to several seconds. The observed trapping behavior is in good quantitative agreement with theoretical expectations.
\end{abstract}

PACS numbers: $32.80 . \mathrm{Pj}$

We report the optical trapping of neutral atoms by the forces of resonance-radiation pressure in a singlebeam optical trap. At the time of the first demonstration of stable optical trapping and manipulation of small dielectric particles ${ }^{1}$ it was predicted that similar effects were possible with atoms. Since then there have been extensive studies of the basic forces of laser light on neutral particles and atoms. ${ }^{2-8}$ The trapping and manipulation of neutral macroscopic dielectric particles from $\sim 30 \mu \mathrm{m}$ to $\sim 25 \mathrm{~nm}$ has been demonstrated. ${ }^{5,9-11}$ With atoms, resonance-radiation forces have been used for atomic deflection, ${ }^{2,12-14}$ the guiding and focusing of atomic beams, ${ }^{15}$ the slowing and stopping of atomic beams, ${ }^{16,17}$ and the three-dimensional cooling of atoms to a temperature of $2.4 \times 10^{-4}$ $\mathrm{K} .{ }^{18,19}$ While there have been many proposals of optical traps for atoms in the last sixteen years, ${ }^{1,2,5,6,8}$ none has been demonstrated previously.

Optical atom traps are difficult to achieve for several reasons. (i) Their potential wells are shallow, typically $10^{-1}-10^{-2} \mathrm{~K}$. (ii) Their volumes, with the exception of alternating-beam scattering-force traps, ${ }^{8,20}$ are quite small. (iii) Once confined within a trap, atoms are heated by the random fluctuations of the light forces and will "boil" out of the trap in a fraction of a second. ${ }^{4}$ The single-beam trap demonstrated here has a well depth $-5 \mathrm{mK}$ and a volume $\sim 10^{-7} \mathrm{~cm}^{3}$. Use of the recently demonstrated "optical molasses"18,19 was crucial to the present experiment. Optical molasses (OM) is formed by the intersection of three pairs of counterpropagating, mutually orthogonal laser beams tuned half a linewidth below a resonance transition. The OM forms a highly viscous medium of photons capable of cooling the sodium atoms used in this experiment to $2.4 \times 10^{-4} \mathrm{~K}$. The dense collection of atoms confined within $\mathrm{OM}$ provides an excellent source with which to load an optical trap. The random-walk motion of the atoms and their long storage time allows atoms to continuously diffuse to the trap surface and be captured. OM was also used to cool atoms within the trap.

The optical trap demonstrated here is the singlebeam gradient-force trap proposed in $1978 .^{3}$ It con- sists of a single strongly focused Gaussian laser beam tuned about $10^{4}$ natural linewidths below resonance. Conceptually it is the simplest of the proposed traps and offers many advantages. It has no standing waves and consequently minimal dipole-force heating. ${ }^{4}$ It is capable of giving deep optical potential wells, localized to a few optical wavelengths, and is ideal for achieving high atomic densities and for optical manipulation of atoms.

The properties of the single-beam trap result from the dominance of the dipole force over the scattering force in a focused Gaussian beam. The scattering force $^{2}$ is due to the spontaneous scattering of photons. Below saturation, it is proportional to the light intensity and points in the direction of the incident light. Quantitatively, $F_{\text {scat }}=(h / \lambda)\left(1 / 2 \tau_{N}\right)[p /(1$ $+p)$ ] where $\tau_{N}$ is the natural lifetime and $p$ is the saturation parameter. Also, $p=\left(I / I_{s}\right)\left[\gamma^{2} / 4\left(\Delta \nu^{2}+\gamma^{2} /\right.\right.$ 4)] where $I_{s}$ is the saturation intensity $\left(20 \mathrm{~mW} / \mathrm{cm}^{2}\right.$ for the $D_{2}$ line), $I=I(r, z)$ is the Gaussian beam intensity, $\Delta \nu$ is the detuning from resonance, and $\gamma=1 / 2 \pi \tau_{N}$ is the natural linewidth. The dipole (or gradient) force, ${ }^{3}$ which arises from the atomic dipole moment induced by the laser field, is proportional to and points in the direction of the intensity gradient. It can be derived from a conservative potential ${ }^{3,4} U$ where $U=(h \Delta \nu / 2) \ln (1+p)$. For tunings below resonance $(\Delta \nu<0)$, the dipole force pushes atoms toward the high-intensity region of the light. This assures the radial stability of the trap. In the axial direction one has the additional complication of the scattering force. However, axial stability can be achieved since the axial gradient force can dominate over the destabilizing influence of the scattering force for tunings well below resonance and for strongly focused beams. The above considerations on the scatteringand dipole-force components on atoms are fully consistent with conservation of momentum in the optical-scattering process. ${ }^{1,4,11}$ The dipole potential $U$ can also be understood in terms of the optical Stark shift of an atom. A laser tuned below resonance lowers the ground-state energy of an atom by an amount $U$ and raises the energy of the excited state by the same 
amount. Since an atom spends most of its time in the ground state its total energy is minimized in the region of the maximum laser intensity. Figure 1(a) shows the potential well along the $z$ axis for three laser detunings. Figure 1(b) shows the axial well depth versus detuning below the sodium $D_{2}$ transition. It is the presence of the axial scattering force which accounts for both the asymmetry of the axial potential well and the fact that the depth of the axial potential is always shallower than the corresponding transverse well depth. The basic principles of single-beam gradient traps fed by visously confined particles were demonstrated $^{11}$ in experiments on the trapping of Rayleigh particles as small as $25 \mathrm{~nm}$ in water.

Damping of the atomic motion in the trap is accomplished by periodically turning the trap off and applying $\mathrm{OM}$ by use of square-wave modulation. ${ }^{21}$ This avoids any interference with the damping process due to the optical Stark shift of the atomic resonance caused by the trap beam, ${ }^{4}$ or interference with the trapping process due to the large fraction of excited atoms caused by the OM beams. If the chopping cycle is short compared with one-half of the oscillation period of an atom in the potential well $(\sim 10 \mu \mathrm{s})$,
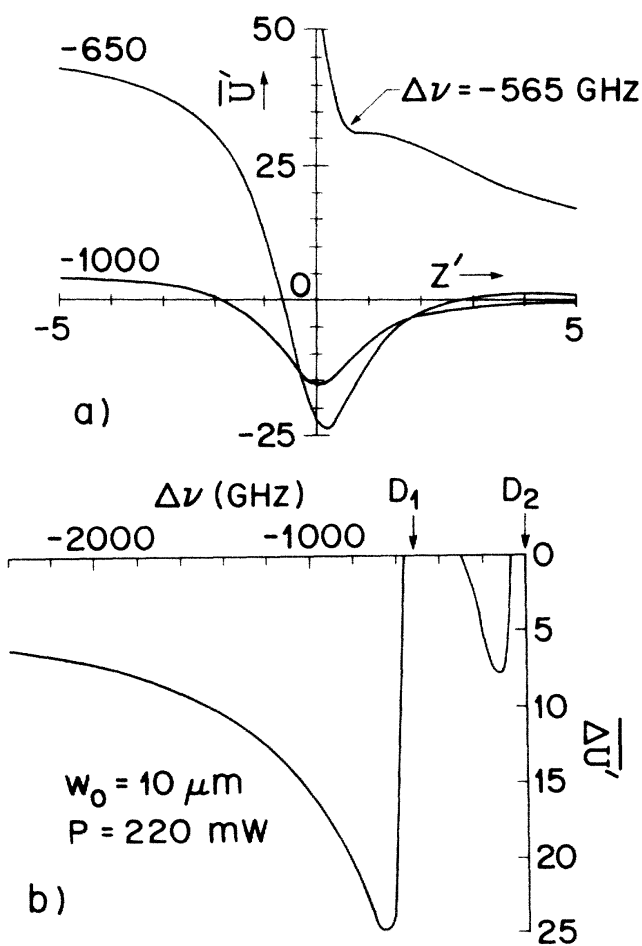

FIG. 1. (a) The normalized, time-averaged axial potential $\bar{U}^{\prime}=2 \bar{U} / h \gamma$ as a function of the axial coordinate $z^{\prime}=z \lambda /$ $\pi w_{0}^{2}$ for $w_{0}=10 \mu \mathrm{m}, P=220 \mathrm{~mW}$, and for three values of trap-laser detuning below the $D_{2}$ resonance line. The calculation uses $I_{s}\left(D_{1}\right)=2 I_{s}\left(D_{2}\right)=40 \mathrm{~mW} / \mathrm{cm}^{2}$. (b) The normalized, time-averaged, potential well depth versus $\Delta \nu$ for $w_{0}=10 \mu \mathrm{m}$ and $P=220 \mathrm{~mW}$. then an atom in the trap does not move much during the chopping cycle and its motion is roughly that of a damped harmonic oscillator with an average well depth of one-half the $\mathrm{cw}$ well depth. In our trap, the heating rate due to the trapping forces is so small that the temperature of the atoms in the trap remains close to the cw OM temperature.

Our experimental apparatus is similar to the apparatus used earlier to demonstrate $\mathrm{OM}^{18,19}$ with the addition of a focused trapping beam nearly parallel to one of the OM beams. Pulses of sodium atoms were created every $0.1-10 \mathrm{~s}$ by evaporating sodium metal with a 10-ns, pulsed, yttrium-aluminum-garnet laser beam. Atoms with a velocity of about $2 \times 10^{4} \mathrm{~cm} / \mathrm{s}$ were slowed over a distance of $8 \mathrm{~cm}$ to about $2 \times 10^{3}$ $\mathrm{cm} / \mathrm{s}$ by the radiation pressure of a frequency-chirped laser beam counterpropagating with the atomic beam. An electro-optic modulator at $856.2 \mathrm{MHz}$ is used to create sidebands $1712.4 \mathrm{MHz}$ apart to prevent optical pumping. ${ }^{18}$ The slowed atoms drift into the OM region where three-dimensional cooling and viscous confinement occurs. The OM-beam spot size is about 1 $\mathrm{cm}$ and the peak intensity is about $20 \mathrm{~mW} / \mathrm{cm}^{2}$ in the relevant sidebands. The optimum tuning of the OM beams $(\sim \gamma / 2$ below resonance) is determined empirically by tuning for maximum storage time. We routinely achieve OM lifetimes of $0.5 \mathrm{~s}$ and densities of $10^{6}$ atoms $/ \mathrm{cm}^{3}$. Temperatures as low as $0.24 \times 10^{-3} \mathrm{~K}$ were previously measured. ${ }^{18}$ The trap laser beam, obtained from a second dye laser, enters the vacuum chamber nearly parallel to one of the OM beams. The linearly polarized trapping beam has a power of about $220 \mathrm{~mW}$ and is focused to a diffraction-limited spot radius $w_{0}=10 \mu \mathrm{m}$.

The optically trapped atoms were detected visually, by video camera, and photographically. Figure 2 (a) is a 0.5-s exposure that shows the fluorescence from atoms in the initial slowing beam, the subsequently formed OM cloud, and atoms collecting in the trap. Figure 2(b) taken later shows the bright spot from trapped atoms which remain in the trap after most of the surrounding $\mathrm{OM}$ atoms have diffused away. The brightness of the fluorescence from the trapped atoms indicates a density much higher than the surrounding cloud of cold atoms. Virtually all of the fluorescence is caused by the OM beams since our trap is tuned far from the atomic resonance.

Several tests were made to confirm trapping: (i) The bright oval-shaped spot coming from trapped atoms occurs only for tunings within the range expected for axial trapping. For example, strong trapping was observed between -570 to at least $-1300 \mathrm{GHz}$ below the $D_{2}$ resonance with the deepest traps occurring at $-650 \pm 25 \mathrm{GHz}$. This agrees well with calculations based on Fig. 1(b). (ii) We observed visually that the lifetime of trapped atoms was longer than for 


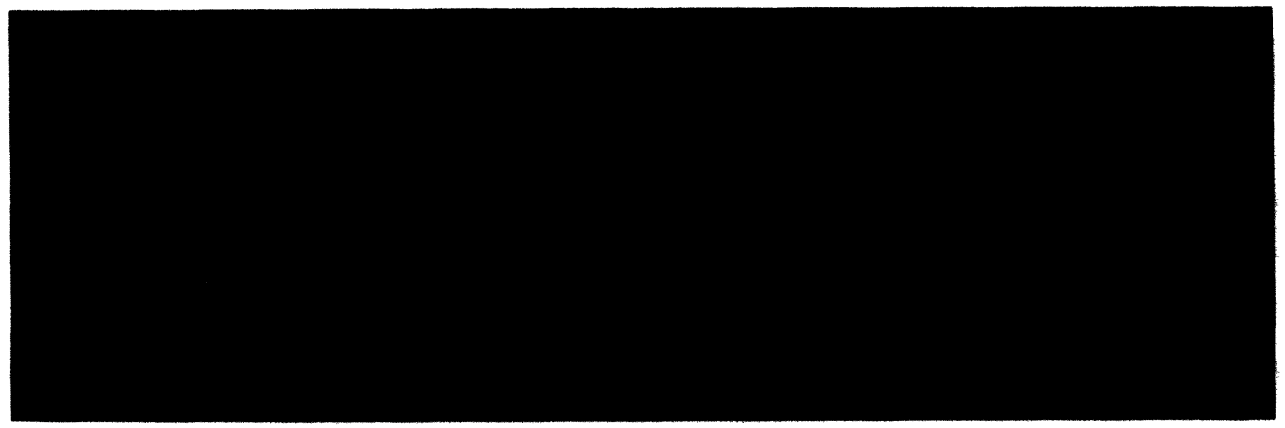

(a)

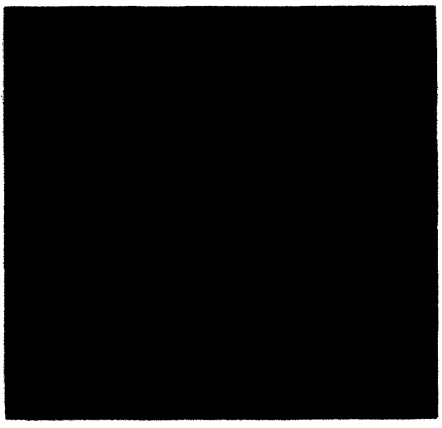

(b)

FIG. 2. (a) Photo showing the collimating nozzle, atomic beam, and atoms confined in OM. The distance from the nozzle to the $\mathrm{OM}$ region is $5 \mathrm{~cm}$. (b) Photo taken after the atomic source and the slowing laser beam have been turned off, showing trapped atoms.

confined $\mathrm{OM}$ atoms. To quantify these observations we analyzed video tapes of the trap decay using a video waveform monitor. Figure 3(a) shows the signal of one horizontal video line through the trap center. The curve $S_{1}$ in Fig. 3(b) shows that atoms confined within only OM decay with a lifetime of about $0.5 \mathrm{~s}$. Curve $S_{2}$ is for trapped atoms plus atoms in OM. The fact that $S_{2}$ is nonzero for more than a second after $S_{1}$ has fully decayed indicates that trapped atoms are longer lived. We surmise that background pressure is limiting the trap decay lifetimes to $\sim 1$ s. Since background gas atoms are at $\sim 300 \mathrm{~K}$ and trapped atoms will be ejected by collisions transferring $<10^{-2} \mathrm{~K}$, we expect a large cross section for ejection of $\sim 10^{-13}$ $\mathrm{cm}^{2}$. (iii) The shape of the trap fluorescence varied with tuning and trap-laser intensity as expected from the calculated axial potential profiles shown in Fig. 1(a). As an example, for strong trapping at -650 $\mathrm{GHz}$ we see a bright oval-shaped spot $\sim 0.5 \mathrm{~mm}$ long, but not fully resolved. As the power is lowered, the oval lengthens and finally a long weak fluorescent streak appears only on the $+z$ side of the trap due to atoms confined transversely by the stronger radial potential well. (iv) Measurements were made on the ef-
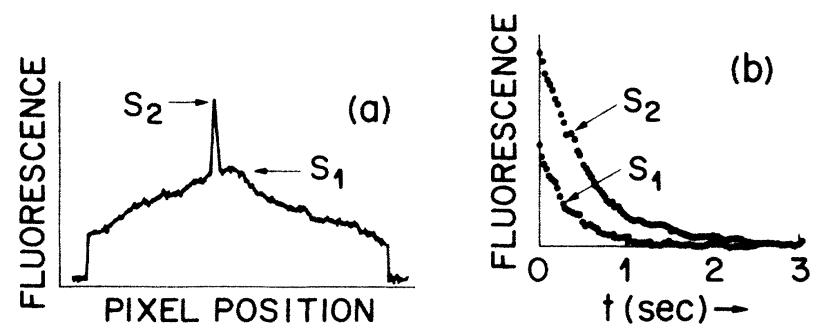

FIG. 3. (a) Video signal on a single horizontal video line through the trap showing scattered light, camera noise, atoms in OM, and a sharp spike due to the fluorescence of the trapped atoms. Data were quantified by use of boxcar integrators to measure the time dependence of the levels $S_{2}$ and $S_{1}$. (b) Time dependence of $S_{2}$ (trap plus OM fluorescence) and $S_{1}$ (OM fluorescence). fect of our varying the chopping period for the trapping and $\mathrm{OM}$ beams. At $220-\mathrm{mW}$ and $-650-\mathrm{GHz}$ tuning, good trapping was obtained for chopping periods between 0.4 and $10.0 \mu \mathrm{s}$. Computer simulations of the radial motion by use of average optical forces shows that the atomic motion becomes unstable for periods longer than $8 \mu \mathrm{s}$. We believe that trapping fails at fast chopping rates as a result of the generation of frequency sidebands, which is detrimental to optical cooling.

A measurement of the trap heating time was made by turning off the OM while keeping the trap on for varying times $(0,5,10,15 \mathrm{~ms})$. At times of $5 \mathrm{~ms}$ the brightness of the trapped atoms decreases by about a factor of 2. This agrees with our calculated trap heating time ${ }^{4}$ of $4 \mathrm{~ms}$.

We can determine the temperature of trapped atoms from the observation that at $-750 \mathrm{GHz}$ strong traps were obtained for 220 and $110 \mathrm{~mW}$ while no trapping was seen for $90 \mathrm{~mW}$. This is done by calculating the intrinsic leakage rate of the trap as a function of power and temperature. The leakage rate is given by the escape-attempt frequency (twice the oscillation frequency of an atom in the trap) times the escape probability, $\exp (-\Delta U / k T)$. To agree with observation, we require intrinsic trap lifetimes of $1 \mathrm{~s}$ or greater for 110 $\mathrm{mW}$ and much less than $1 \mathrm{~s}$ for $90 \mathrm{~mW}$. The result is, $0.6 \leqslant k T / h \gamma \leqslant 0.8$; thus the trapped-atom temperature is remarkably close to the quantum limit for atoms in $\mathrm{OM},{ }^{18} k T=\frac{1}{2} h \gamma=240 \mu \mathrm{K}$. The intrinsic trap lifetimes corresponding to $k T=0.7 h \gamma$ are $0.12 \mathrm{~s}$ for $90 \mathrm{~mW}, 1.0 \mathrm{~s}$ for $110 \mathrm{~mW}$, and $3.7 \times 10^{4} \mathrm{~s}$ for 220 $\mathrm{mW}$. The inferred temperature for the trapped atoms allows us to calculate the fraction of the trapping volume in which the atoms reside. For a detuning of $-650 \mathrm{GHz}$, a trap beam power of $220 \mathrm{~mW}$, and an atomic temperature of $0.7 \mathrm{h \gamma}$, the atoms are confined within a cylinder of length $210 \mu \mathrm{m}$ and diameter of 2.5 $\mu \mathrm{m}$, giving a volume of $1 \times 10^{-9} \mathrm{~cm}^{3}$.

We deduced the number of atoms in the trap and 
their density in several independent ways. From an absolute calibration of the detection system (zoom lens, video camera, and waveform monitor combination), we conclude that our best trap signals are due to about 500 atoms. Based on the confinement volume of $1 \times 10^{-9} \mathrm{~cm}^{3}$, the corresponding trapped-atom density is $5 \times 10^{11} \mathrm{~cm}^{-3}$. An accurate estimate of the trapped-atom density is also obtained by noting that the signal from the trapped atoms is roughly equal to the signal from the atoms in OM. Using the f-stop number of the video-camera lens and assuming a $\mathrm{OM}$ radius of $0.5 \mathrm{~cm}$, we estimate that the average trap density is $1.5 \times 10^{6}$ times the OM density. A OM density of $5 \times 10^{5} \mathrm{~cm}^{-3}$ yields a trap density of $8 \times 10^{11}$ $\mathrm{cm}^{-3}$, reasonably consistent with the previous estimate.

Finally we note that the collection of trapped atoms could be moved easily at speeds on the order of $1 \mathrm{~cm} / \mathrm{s}$ by manually scanning the location of the trap focal spot.

In summary, optical trapping of neutral atoms has been observed in good quantitative agreement with theoretical expectations. We have achieved ordersof-magnitude higher densities of trapped atoms and lower temperatures than reported previously using magnetic neutral-atom traps ${ }^{22}$ and electrodynamic ion traps. ${ }^{23,24}$ It may be possible to increase the density by $\geq 10^{4}$ and to decrease the temperature to $\leq 10^{-6} \mathrm{~K}$. This would involve tighter beam focusing, additional cooling of OM with narrower linewidth transitions, or possibly beam-expansion schemes. ${ }^{25}$ Confinement and spatial manipulation of cold trapped atoms to dimensions less than the optical wavelength are also possible. These capabilities should prove useful in studies of Bose and Fermi gas statistics and other interactions at high atomic density, atom-atom collisions, atom-surface interactions, atom diffraction and tunneling, molecular formation using individual atoms, modifications of atomic spontaneous-emission lifetimes and other collective effects, spectroscopy of the trap energy levels, and, possibly, high-resolution spectroscopy and atomic time standards.

The authors thank J. P. Gordon for valuable discussions.

\footnotetext{
${ }^{1}$ A. Ashkin, Phys. Rev. Lett. 24, 156 (1970).
}

${ }^{2}$ A. Ashkin, Phys. Rev. Lett. 25, 1321 (1970).

${ }^{3}$ A. Ashkin, Phys. Rev. Lett. 40, 729 1978).

4J. P. Gordon and A. Ashkin, Phys. Rev. A 21, 1606 (1980).

${ }^{5}$ A. Ashkin, Science 210, 1081 (1980).

${ }^{6}$ V. Letokhov and V. Minogin, Physics Rep. 73, 3 (1981).

${ }^{7}$ Laser-Cooled and Trapped Atoms, edited by W. D. Phillips, Progress in Quantum Electronics Vol. 8 (Pergamon, New York, 1984).

${ }^{8}$ A. Ashkin, Opt. Lett. 9, 454 (1984).

${ }^{9}$ G. Roosen, Can. J. Phys. 57, 1260 (1979).

${ }^{10}$ A. Ashkin and J. M. Dziedzic, Phys. Rev. Lett. 54, 1245 (1985).

${ }^{11}$ A. Ashkin, J. M. Dziedzic, J. E. Bjorkholm, and Steven Chu, Opt. Lett. 11, 288 (1986).

${ }^{12}$ R. Schieder, H. Walther, and L. Wöste, Opt. Commun. 5, 337 (1972).

13P. Jacquinot, S. Liberman, J. L. Picque, and J. Pinard, Opt. Commun. 8, 163 (1973).

14J. E. Bjorkholm, R. R. Freeman, and D. B. Pearson, Phys. Rev. A 23, 491 (1981).

15J. E. Bjorkholm, R. R. Freeman, A. Ashkin, and D. B. Pearson, Phys. Rev. Lett. 41, 1361 (1978).

16J. Prodan, A. Migdall, W. D. Phillips, I. So, and H. Dalibard, Phys. Rev. Lett. 54, 992 (1985).

${ }^{17}$ E. Ertmer, R. Blatt, J. L. Hall, and M. Zhu, Phys. Rev. Lett. 54, 996 (1985).

${ }^{18}$ Steven Chu, L. Hollberg, J. E. Bjorkholm, Alex Cable and A. Ashkin, Phys. Rev. Lett. 55, 48 (1985).

${ }^{19}$ Steven Chu, J. E. Bjorkholm, A. Ashkin, L. Hollberg, and Alex Cable, "Methods of Laser Spectroscopy" (Plenum, New York, to be published).

${ }^{20} \mathrm{We}$ have recently learned of a newly proposed scattering-force optical trap that can have volumes and well depths comparable with alternating-beam scattering-force traps: D. E. Pritchard, private communication, and D.E. Pritchard et al., preceeding Letter [Phys. Rev. Lett. 57, 310 (1986)].

21J. Dalibard, S. Reynaud, and C. Cohen-Tannoudji, Opt. Commun. 47, 395 (1983).

22 A. L. Migdall, J. V. Prodan, W. D. Phillips, T. H. Bergeman, and H. J. Metcalf, Phys. Rev. Lett. 54, 2596 (1985).

${ }^{23}$ D. J. Wineland, W. M. Itano, J. C. Bergquist, J. J. Bollinger, and H. Hemmate, Prog. Quantum Electron. 8, 139 (1984).

${ }^{24}$ W. Nagourney, G. Janik, and H. Dehmelt, Proc. Natl. Acad. Sci. U.S.A. 80, 643 (1983).

${ }^{25}$ S. Chu, J. E. Bjorkholm, A. Ashkin, J. P. Gordon, and L. W. Hollberg, Opt. Lett. 11, 73 (1986). 

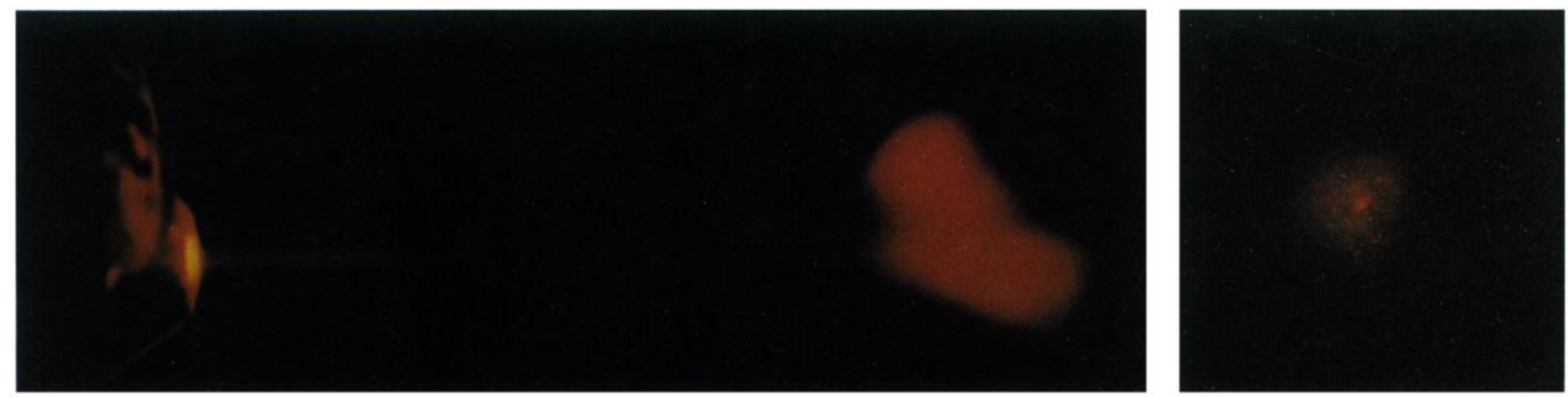

(a)

(b)

FIG. 2. (a) Photo showing the collimating nozzle, atomic beam, and atoms confined in OM. The distance from the nozzle to the $\mathrm{OM}$ region is $5 \mathrm{~cm}$. (b) Photo taken after the atomic source and the slowing laser beam have been turned off, showing trapped atoms. 